\title{
PLAN DE ACCIÓN TUTORIAL PARA PREVENIR EL ACOSO ESCOLAR TRANSFÓBICO EN LA ADOLESCENCIA
}

\author{
Tara Sánchez Sánchez \\ Universidad de Las Palmas de Gran Canaria \\ taraasanchez@hotmail.com \\ María del Pilar Etopa Bitata \\ Universidad de Las Palmas de Gran Canaria \\ pilar.etopa@ulpgc.es
}

Recepción Artículo: 03 agosto 2021 Admisión Evaluación: 03 agosto 2021 Informe Evaluador 1: 17 agosto 2021 Informe Evaluador 2: 29 agosto 2021 Aprobación Publicación: 01 septiembre 2021

\section{RESUMEN}

Diversas investigaciones sobre el acoso escolar transfóbico en España corroboran que el 46,2\% del acoso que sufren las personas trans* ocurre en los centros educativos, convirtiéndose en el segundo contexto donde más acoso experimenta este colectivo (Devís-Devís et al., 2017). Por tanto, a pesar de la implementación en el año 2014 de la Ley de Igualdad LGTBI por parte de ciertas comunidades autónomas, hoy en día, aparece en las aulas un 58\% de casos de acoso transfóbico en todas las etapas educativas, observándose un 37\% de casos en la etapa de secundaria (FELGTB, 2019a). En cuanto a la tipología de las agresiones, pese a la variabilidad, las investigaciones hacen referencia a las agresiones verbales como las más frecuentes (Devís-Devís et al., 2017; FELGTB, 2019a; FELGTB, 2019b; Martín et al., 2019), pero las y los menores trans* también sufren agresiones físicas, aislamiento social, ciberacoso y acoso sexual (FELGTB, 2019a). Por ello, cuando el acoso transfóbico se prolonga en el tiempo afecta a la estabilidad psicológica de la víctima, elevando el riesgo de suicidio que, según los estudios, afecta al $20 \%$ de los chicos y las chicas trans*. A este respecto, se destaca la importancia de afrontar esta realidad en las instituciones educativas, puesto que, tanto los acontecimientos sociales que vivimos día a día como las cifras generales de diversas investigaciones estatales muestran que los casos de transfobia han crecido exponencialmente, sucediendo más de la mitad de ellos en los centros educativos. Dada esta alarmante situación, surge la necesidad de elaborar un programa de intervención para, a través del Plan de Acción Tutorial, prevenir el acoso escolar hacia el alumnado trans* en la etapa de Educación Secundaria; formando a jóvenes más concienciados, empáticos y asertivos que favorezcan la creación de entornos seguros e inclusivos, impulsando el cambio social.

Palabras clave: acoso escolar transfóbico; alumnado trans*; Educación Secundaria; Plan de Acción Tutorial. 


\section{PLAN DE ACCIÓN TUTORIAL PARA PREVENIR EL ACOSO ESCOLAR TRANSFÓBICO EN LA ADOLESCENCIA}

\section{ABSTRACT}

Tutorial action plan to prevent transphobic bullying in adolescent. Various studies on transphobic bullying in Spain corroborate that $46.2 \%$ of the bullying suffered by trans* people occurs in educational centers, making it the second context in which this group experiences the most bullying (Devís-Devís et al., 2017). Therefore, despite the implementation in 2014 of the LGTBI equality law by certain autonomous communities, today, $58 \%$ of cases of transphobic bullying appear in the classrooms in all educational stages, observing $37 \%$ of cases in secondary school (FELGTB, 2019a). Regarding the typology of aggressions, despite the variability, research refers to verbal aggressions as the most frequent (Devís-Devís et al., 2017; FELGTB, 2019a; FELGTB, 2019b; Martín et al., 2019), but trans * minors also suffer physical attacks, social isolation, cyberbullying and sexual harassment (FELGTB, 2019a). Therefore, when transphobic bullying is prolonged over time, it affects the psychological stability of the victim, increasing the risk of suicide, which, according to studies, affects $20 \%$ of trans * boys and girls. In this regard, the importance of facing this reality in educational institutions is highlighted, since both the social events that we experience every day and the general figures from various state investigations show that cases of transphobia have grown exponentially, with more happening of half of them in educational centers. Given this alarming situation, the need arises to develop an intervention program to, through the Tutorial Action Plan, prevent bullying towards trans * students in the Secondary Education stage; training more conscientious, empathetic and assertive young people who favor the creation of safe and inclusive environments, promoting social change.

Keywords: transphobic bullying; trans * students; Secondary education; Tutorial Action Plan

\section{ANTECENDENTES}

Una de las investigaciones realizadas sobre el acoso transfóbico en el panorama nacional revela que pertenecer al colectivo LGTBIQ+ es el tercer motivo de acoso y discriminación en España, posicionando a las Islas Canarias como la cuarta comunidad autónoma con más delitos de odio registrados en el año 2019 (FELGTB, 2019b).

El índice de acoso que sufren las personas trans* es más elevado que el de las personas cisexuales, por lo que formar parte de este colectivo se convierte en un factor de riesgo (Pérez et al., 2021). Además, en comparación con el resto del colectivo LGTBIQ+, las personas trans* están expuestas a un mayor número de agresiones (Martín et al., 2019). Lo mismo ocurre en el ámbito educativo, siendo el acoso transfóbico una de las principales causas de acoso escolar (FELGTB, 2019b). Asimismo, se debe tener en cuenta que el $41 \%$ del alumnado trans* admite no considerarse aceptado socialmente (Martín et al., 2019).

Resulta estremecedora la aportación de Martín et al. (2019) que hace referencia al incremento en las tasas de acoso escolar que vivencia el colectivo LGTBIQ+, considerando sus distintas manifestaciones, al igual que el miedo, la carencia de información y la desprotección que siente este colectivo por parte de sus familias y de los docentes. Por esta razón, las y los jóvenes trans* deben contar con redes de apoyo que consigan evitar el intento de suicidio, siendo de vital importancia el apoyo familiar (Pichardo y De Stéfano, 2015).

Otro aspecto que se debe tener en consideración es que en la mayoría de los centros se ha trabajado tanto la diversidad como el acoso escolar, pero, aun así, se siguen observando elevadas cifras de acoso escolar transfóbico. Es más, si se analiza la evolución de este tipo de acoso desde el año 2015 hasta el año 2018, hay un incremento del 7\%, adquiriendo, por tanto, una mayor incidencia, lo cual resulta preocupante (Martín et al., 2019).

\section{OBJETIVOS}

El objetivo general es concienciar y sensibilizar a las y los adolescentes acerca de las realidades trans* para prevenir el acoso escolar hacia el alumnado trans* en esta etapa, además de incidir en el resto de la comunidad educativa contribuyendo al cambio social. 


\section{PARTICIPANTES}

El programa de intervención se llevará a cabo a través del Plan de Acción Tutorial, comenzando con el alumnado de 1ํㅡㄹ ESO concienciándoles acerca de las realidades trans* y mostrándoles la diversidad como una fuente de enriquecimiento.

\section{MÉTODO}

Se utilizarán instrumentos de evaluación con la intención de conocer la evolución de los conocimientos del alumnado.

Con este fin, se elaborará un cuestionario que será cumplimentado por las y los estudiantes, tanto al comienzo (pretest) como al final de la intervención (postest). Esto nos indicará el nivel inicial del alumnado para, posteriormente, comprobar el alcance de su aprendizaje, significativo y didáctico, los objetivos propuestos al inicio de la intervención.

Durante el desarrollo del programa de intervención se recogerán los detalles más significativos de cada sesión en un diario, haciendo uso de un instrumento de observación directa. También se tendrán en cuenta las producciones del alumnado, así como el desarrollo del taller dirigido a las familias, pues las y los jóvenes plasmarán en él todo lo aprendido.

A modo de feedback y como cierre evaluativo, se proporcionará un cuestionario compuesto por preguntas abiertas, para conocer el punto de vista y grado de satisfacción del alumnado respecto al trabajo realizado, lo cual servirá como medio de perfeccionamiento para la siguiente implementación del programa, ya sea en el mismo curso u otros superiores.

En la evaluación del programa participarán el Departamento de Orientación, las y los tutores y las familias, para conocer si el programa se ha ajustado a las necesidades y demandas de las y los participantes. Para ello, entre las diversas aportaciones, se tendrá en consideración si las actividades se adecúan al nivel del alumnado, si la temporalización y la ubicación de la puesta en práctica ha favorecido el aprendizaje, formando a personas que posean valores esenciales, como el respeto, la tolerancia, la solidaridad y la empatía o si es preciso realizar cambios para conseguir una mejor ejecución del mismo, subsanando las dificultades que hayan podido surgir durante el abordaje de esta temática.

\section{RESULTADOS}

\section{Módulo 1: ¡Una mirada a las realidades diversas!}

En este primer módulo se desarrollarán los términos relacionados con la diversidad sexual, de modo que el alumnado parta de una comprensión global de los conocimientos básicos, en conjunción con el desarrollo de un pensamiento crítico al abordar esta temática, desmontando algunos mitos sobre el tema de manera didáctica. 


\section{Tabla 1
Nuestro diccionario sobre diversidad sexual}

Descripción: Con el fin de que el alumnado conozca la terminología pertinente para desarrollar este tema y de este modo conseguir un aprendizaje significativo, es esencial partir de los conocimientos que ya posee e ir abordando, paulatinamente, la nueva información.

Esta primera sesión se desarrollará como una toma de contacto en la cual el alumnado deberá responder, de manera individual, un cuestionario inicial, tras el cual se visualizará el vídeo “¿Qué es la diversidad sexual?” para tratar de explicar los conceptos básicos necesarios para comprender y conocer las realidades trans*.

Una vez finalizado el vídeo, se invitará al alumnado a reflexionar sobre la terminología abordada para, entre todas y todos, crear nuestro diccionario sobre la diversidad sexual, anotando las diversas aportaciones en un mural.

\section{Objetivos}

- Averiguar los conocimientos previos que posee el alumnado sobre las realidades trans*.

- Conocer los términos relacionados con la diversidad sexual.

\section{Acceso a los recursos utilizados:}

Carki Productions. (25 de julio de 2017). ¿Qué es la diversidad sexual? Explicación fácil. [Vídeo]. Youtube.

https://www.youtube.com/watch?v=1QbTZYiQ6BA

Puesta en práctica: Esta actividad se implementó en un grupo de $1^{\circ} \mathrm{ESO}$.

Entre las preguntas más frecuentes sobre los cambios puberales se encontraba la menstruación y el desarrollo de los genitales, mientras que sobre la terminología surgieron dudas en torno a la orientación sexual de las personas trans*, confundiendo, en algunos casos, identidad de género y orientación sexual.

Otro aspecto a destacar es el interés y respeto que mostraron durante el abordaje de este tema, mostrándose muy participativos.

Fuente: Elaboración Propia. 


\section{Tabla 2 \\ Bingo de conceptos}

Descripción: En esta actividad, se realizará un bingo para repasar la terminología relacionada sobre la diversidad sexual. A través de esta actividad se procederá a: resolver dudas que hayan podido surgir, clarificar términos, reforzar conocimientos y situar a personas que hayan podido faltar a la actividad anterior.

Para ello, se dividirá al alumnado en grupos de $4 \mathrm{y}$, cada grupo trabajará, de manera colaborativa, con un tablero del bingo.

El orientador o la orientadora irá diciendo en voz alta la definición de los conceptos, mientras que las y los estudiantes deberán ir marcando la palabra correspondiente en el tablero. El grupo que primero finalice tendrá que decir: ¡BINGO!

Al finalizar la actividad, se preguntará a las y los adolescentes si saben más términos asociados a las realidades trans*, dejando tiempo para que cada grupo busque en internet información al respecto y, posteriormente, hacer una puesta en común que propicie la incorporación de nuevos términos al diccionario.

\section{Objetivos}

- Clarificar la terminología abordada sobre la diversidad sexual.

- Acercar al alumnado al conocimiento de las realidades trans*.

Fuente: Elaboración Propia.

\section{Tabla 3}

\section{Desmontando mitos}

Descripción: Con el desarrollo de esta actividad, se pretende que las y los jóvenes muestren su concepción con respecto a una serie de afirmaciones que realizará el orientador o la orientadora para poder llegar de este modo y a través de una reflexión conjunta a ciertas conclusiones.

Para ello, nos dirigiremos a una zona al aire libre, donde el grupo se repartirá en el espacio del modo que prefieran. Allí se entregarán tres palas a cada persona (una verde, otra roja y otra naranja), las cuales simbolizarán el sí, el no y el no sé respectivamente. Es entonces cuando se dispondrán a hacer una serie de afirmaciones a las que, transcurridos unos minutos de reflexión, el alumnado tendrá que responder utilizando las palas, tras lo cual, y abogando por un pensamiento crítico, se les preguntará el porqué de sus respuestas y se desarrollará un debate con la información expuesta. Después de dicho intercambio de opiniones e información, se volverá a realizar la afirmación, una vez más, posibilitando que los chicos y las chicas puedan cambiar o no la opinión expresada con anterioridad.

Las afirmaciones que se plantearán son las siguientes:

- Algunas personas no se identifican con el sexo asignado al nacer.

- Las personas trans* están atrapadas en un cuerpo equivocado.

- Una persona trans* termina su proceso cuando se opera.

- Ser trans* es una elección, opción y/o capricho.

- Los chicos y las chicas no tienen suficiente madurez para saber que son trans*.

- Ser trans* es lo mismo que homosexual y/o travesti.

- La transexualidad es una enfermedad y tiene cura.

- El colectivo LGTBQ+ ha alcanzado la plena igualdad.

- No deberían existir etiquetas, todos somos personas. 
personas trans* sean capaces de poner de manifiesto el afloramiento de los sentimientos y las emociones, llegando a empatizar con el colectivo.

Tabla 4

Ponte en mi piel

Descripción: Para comenzar este módulo es necesario sensibilizar al alumnado, por lo que se pretende que cada persona sea capaz de experimentar diferentes emociones que, posteriormente, les ayuden a comprender cómo se sienten las personas trans* cuando son juzgadas, rechazadas y estigmatizadas por la sociedad. De esta manera será más sencillo concienciar a las y los adolescentes, queriendo a su vez que sean empáticos con este colectivo. Así pues, la sesión se dividirá en dos partes.

En la primera parte, se irá nombrando a cada persona para que cuente algún aspecto característico que la defina y/o forme parte de ella. De modo que, haciendo uso de la información obtenida, el resto de sus compañeros y compañeras deberán ir realizando diversas preguntas, cuestionando así la veracidad de la información aportada por el o la participante. Esta situación hará que vivencien ese sentimiento que muchas veces tienen las personas trans* al ser juzgados o puestos en duda por el resto de la sociedad. Respecto a la segunda parte, se procurará que las y los estudiantes empaticen con el colectivo trans*. Para ello, escribirán en un papel de manera anónima ciertas situaciones en las que hayan vivenciado algún tipo de rechazo por parte de alguna persona, al igual que expresarán cómo se sintieron en ese momento y qué necesitaban. Dichos relatos serán compartidos en voz alta por el orientador o la orientadora con el resto de los alumnos y las alumnas.

Finalmente, se hará una reflexión de la sesión, mediante la cual cada persona contará cómo se ha sentido cuando sus iguales, siendo conocedores solamente de ciertos detalles, les juzgaban o, incluso, dudaban de la credibilidad de su testimonio. Las aportaciones del alumnado se irán apuntando en la aplicación "Wordle" para crear una nube de palabras que les ayude a visualizar de manera global cómo se pueden sentir las personas trans* al generarse situaciones similares por formar parte del colectivo.

\section{Objetivos}

- Objetivo 7: Sensibilizar al alumnado sobre la discriminación y el rechazo que sufren las personas trans*.

- Objetivo 8: Concienciar a las y los adolescentes sobre la importancia del respeto, la empatía, la tolerancia y la comprensión.

Fuente: Elaboración Propia.

\section{Tabla 5}

3 generaciones

Descripción: En esta actividad, el alumnado visualizará la película "3 generaciones" (2015), donde la directora, Gaby Dellal, quiso mostrar la realidad que vivencian muchas familias en la actualidad. Así pues, fruto de los distintos relatos que fue conociendo, surgió una historia sobre tres generaciones, a través de la cual se aborda tanto la diversidad familiar como la diversidad sexual.

El protagonista, Ray, es un adolescente trans* que decide someterse a una operación de reasignación de sexo, para ello necesita el consentimiento legal de ambos progenitores, siendo un obstáculo en su tránsito, dado que este vive con su madre soltera y no tiene contacto con su padre biológico. Este trámite burocrático hace que 
deban encontrar al padre biológico y lidiar con los fantasmas del pasado de su madre. A su vez, la abuela del menor no acepta que ahora tiene un nieto, debido a que considera que, al igual que ella, es homosexual en vez de trans*. Por tanto, durante este proceso, los miembros de la familia tendrán que confrontar sus identidades y mantenerse unidos para conseguir la aceptación y el entendimiento a través del acompañamiento. Se debe mencionar que en la película se observan conductas discriminatorias, tanto por parte de familiares como de su grupo de iguales, siendo solventadas gracias al apoyo y comprensión de sus seres queridos.

Posteriormente, el alumnado, después de reflexionar acerca de esta realidad, responda a una serie de preguntas, fomentando así el espíritu crítico. Para ello, el tutor o la tutora colgará en el classroom el enlace del formulario de google que contiene las siguientes cuestiones:

- ¿Cómo hubieses actuado si estuvieses en la situación de Ray?

- ¿Qué parte de la película te ha gustado más? ¿Por qué?

- ¿Qué opinas acerca de la familia de Ray?

- ¿Cómo crees que se sintió Ray ante la violenta situación que sufrió en la calle?

- ¿Qué consideras que podría hacer el entorno de Ray para ayudarlo?

- ¿Por qué consideras que los hermanos pequeños de Ray aceptaron y comprendieron mejor el tránsito que las personas adultas?

- Define a Ray utilizando tres palabras.

- Imagina que Ray es tu mejor amigo y te cuenta que quiere transitar ¿Qué le dirías?

\section{Objetivos}

- Fomentar una visión crítica sobre la situación que suelen vivenciar las y los adolescentes trans*.

- Visibilizar la realidad de las personas trans*, fomentando el respeto a la diversidad.

- Sensibilizar acerca de la diversidad familiar.

- Suscitar la empatía hacia las personas trans*.

Fuente: Elaboración Propia.

\section{Tabla 6}

\section{La importancia de un buen acompañamiento}

Descripción: En esta actividad, se pretende acercar al alumnado a la realidad de las personas trans*, concienciándoles de la importancia que tiene propiciar y llevar cabo un buen acompañamiento.

Para su desarrollo, se contará con la colaboración de personas trans* que narrarán su experiencia, tanto en el ámbito escolar como familiar, donde algunas personas han experimentado el apoyo, la comprensión y el acompañamiento de su entorno, mientras que otras han vivenciado la exclusión y el rechazo. Por lo tanto, se expondrán aquellos aspectos positivos y negativos que han repercutido en la vida de cada persona y comentarán qué necesitaban en cada momento.

Finalmente, el alumnado dispondrá de un tiempo para preguntar aquellas cuestiones que considere pertinente. 


\section{PLAN DE ACCIÓN TUTORIAL PARA PREVENIR EL ACOSO ESCOLAR TRANSFÓBICO EN LA ADOLESCENCIA}

\section{Objetivos}

- Acercar al alumnado al conocimiento de las realidades trans*.

- Reflexionar sobre las repercusiones que tienen las situaciones negativas en la vida de las personas trans*.

- Visibilizar el acoso escolar transfóbico.

Fuente: Elaboración Propia.

\section{Módulo 3: ¡La diversidad nos enriquece!}

Este tercer módulo desea mostrar y visibilizar las diversas realidades existentes con el fin de prevenir la transfobia concienciando a toda la comunidad educativa, siendo los alumnos y las alumnas propulsores del cambio.

\section{Tabla 7}

Conocemos referentes trans *

Descripción: En esta actividad, se dividirá al alumnado en pequeños grupos para que busquen, haciendo uso de sus móviles y las tablets del centro educativo, referentes trans* en el mundo de la cultura, del deporte, de la política, del arte, de las ciencias, etc. En otras palabras, personas trans* que llamen su atención y cuya historia les sorprenda, ya que las nuevas generaciones necesitan apoyarse y verse reflejados en personas que sean ejemplo de lucha y superación.

Una vez encontradas, deberán elaborar una pequeña exposición en la cual explicarán porqué les resultan llamativas y si ha cambiado algo su punto de vista respecto a la realidad trans*, así como aquellos aspectos que consideren relevantes. Asimismo, posteriormente, tendrán que realizar una puesta en común con el resto de la clase.

Tras finalizar las exposiciones, se fomentará un debate sobre qué es lo que más les ha sorprendido o llamado la atención, si opinan que hay suficientes referentes o no, si creen que a lo largo de la historia se ha podido llegar lejos siendo trans* y cómo se podría visibilizar más esta realidad. Todo ello argumentando las diferentes respuestas.

\section{Objetivos}

- Visibilizar a referentes trans*, mostrando sus historias y/o experiencias.

Fuente: Elaboración Propia.

Tabla 8

Taller acerca de las realidades trans * con las familias

Descripción: Esta actividad consistirá en la realización de un taller que llevará a cabo el alumnado destinado a las familias, supervisado por el orientador o la orientadora. Todo ello para que las y los jóvenes sean los transmisores del aprendizaje adquirido y las familias se muestren más participativas y receptivas, dado que sus hijos e hijas serán quienes les aporten la información.

Para ello, en pequeños grupos, se encargarán de una de las actividades trabajadas para, de forma creativa, preparar una exposición dirigida a las familias, acercándolos a esta realidad. Podrán realizar una obra de teatro con títeres, un vídeo creado por el grupo donde se visualicen situaciones de discriminación, rechazo y aislamiento, una historia, un debate, etc. Cabe destacar que, las y los estudiantes contarán con una sesión de 45 minutos para preparar su presentación, con ayuda, tanto de la o el tutor como del orientador u orientadora las diferentes actividades. 


\section{Objetivos}

- Conocer los términos relacionados con la diversidad sexual.

- Desmontar ciertos mitos que forman parte de la sociedad.

- Visibilizar la realidad de las personas trans*, fomentando el respeto a la diversidad.

- Suscitar la empatía hacia las personas trans*.

- Promover la participación y colaboración de las familias en el proceso de enseñanza-aprendizaje.

Fuente: Elaboración Propia.

Tabla 9

¡Visibilizamos las realidades trans*!

Descripción: Con el objetivo de concienciar al mayor número de personas y aprovechando que el 31 de marzo es el Día Internacional de la Visibilidad Trans, el alumnado elaborará carteles, haciendo uso de la información obtenida a lo largo del programa. Además, para completar y/o profundizar en la temática, podrán utilizar los dispositivos móviles o las tablets que hay en el aula.

De esta forma, en pequeños grupos y dando rienda suelta a su creatividad, mostrarán, en el vestíbulo principal del centro educativo, la diversidad de las realidades existentes en las pancartas realizadas. Dado que, visibilizando y concienciando se pretende prevenir la transfobia.

Para finalizar la actividad, las y los estudiantes deberán responder individualmente a un cuestionario compuesto por las mismas preguntas que en el cuestionario inicial, lo que contribuirá a la evaluación del aprendizaje de las y los adolescentes y, por consiguiente, constatará (o no) la eficacia de la propuesta de intervención. Además, se les entregará un documento con varias preguntas para conocer el grado de satisfacción de las y los participantes, así como su experiencia en el mismo.

\section{Objetivos}

- Visibilizar la realidad de las personas trans*, fomentando el respeto a la diversidad.

- Comprobar la evolución del alumnado respecto a las realidades trans*.

- Descubrir el grado de satisfacción de las y los jóvenes tras el desarrollo de la intervención.

Fuente: Elaboración Propia.

\section{CONCLUSIONES}

Para garantizar una respuesta educativa que se adecue a las necesidades y demandas existentes en los centros educativos con respecto a las realidades trans ${ }^{*}$, se considera necesario tener en cuenta la normativa vigente y crear acciones concretas que impliquen a toda la comunidad educativa para conseguir una escuela libre de transfobia. De tal manera que, en todas las etapas educativas, se trabajen, tanto a través del currículo como de manera transversal, actividades que acerquen al alumnado a las realidades trans* mediante un enfoque positivo, sin olvidar fomentar el espíritu crítico de las y los estudiantes en lo relacionado a la igualdad, la diversidad y la discriminación. En consecuencia y con el fin de posibilitar todo ello, es necesario que el profesorado reciba una formación y sensibilización que contribuya en el abordaje de esta realidad (Platero, 2014).

Puche et al. (2013) ofrecen una serie de recomendaciones para trabajar las realidades trans* en la etapa de 


\section{PLAN DE ACCIÓN TUTORIAL PARA PREVENIR EL ACOSO ESCOLAR TRANSFÓBICO EN LA ADOLESCENCIA}

Educación Secundaria. En este sentido, las y los docentes pueden aprovechar el Plan de Acción Tutorial para concienciar, visibilizar y sensibilizar a las y los jóvenes sobre las realidades trans*; además de contar con la colaboración de las asociaciones para impartir charlas que proporcionen referentes trans ${ }^{\star}$ y propicien a la comunidad educativa recursos y herramientas sobre el tema en cuestión. Otra propuesta sería aprovechar las distintas asignaturas para incluir contenidos específicos, de tal forma que el alumnado comenzara a comprender la realidad trans* como una parte más de la diversidad. A su vez, el centro educativo debería promover la formación del equipo de mediación para detectar posibles casos de transfobia e intentar erradicarla, pudiendo acudir cualquier alumno 0 alumna para abordar cualquier cuestión relacionada con la diversidad sexual. Todo ello, debe estar reflejado en los documentos institucionales del centro, dado que, en ellos, se deben recoger diferentes acciones que sirvan para prevenir el acoso escolar transfóbico, al mismo tiempo que protocolos de actuación establecidos para intervenir en dicha situación (Pichardo et al., 2015).

Todas las acciones que se lleven a cabo deben favorecer un clima seguro y de confianza, donde el alumnado trans* se sienta apoyado y comprendido. Para ello, es fundamental mantener una escucha activa y una actitud de acompañamiento, teniendo en cuenta los miedos que tiene cada persona y, por consiguiente, lo que necesita de la comunidad educativa (De Stéfano y Pichardo, 2016).

En definitiva, la información, la formación y el respeto a las realidades trans* son fundamentales para conseguir una escuela inclusiva y, por tanto, igualitaria, donde las y los adolescentes trans ${ }^{*}$ no se vean enmarcados en estructuras binarias y conciban el centro educativo como un espacio seguro donde poder desarrollar su identidad tanto personal como de género (Gavilán, 2016).

\section{REFERENCIAS BIBLIOGRÁFICAS}

Devís-Devís, J., Pereira-García, S., Valencia-Peris, A., Fuentes-Miguel, J., López-Cañada, E., \& PérezSamaniego, V. (2017). Harrassment patterns and risk profile in Spanish trans persons. Journal of Homosexuality, 64(2), 239-255. http://dx.doi.org/10.1080/00918369.2016.1179027

Federación Estatal de Lesbianas, Gais, Trans y Bisexuales. (2019a). Jóvenes trans en el sistema educativo. https://felgtb.org/wp-content/uploads/2020/09/Informe-menores-trans_FELGTB_2019.pdf

Federación Estatal de Lesbianas, Gais, Trans y Bisexuales. (2019b). Informe de delitos de odio 2019. https://felgtb.org/wp-content/uploads/2020/06/INFORME_DELITOSDEODI02019.pdf

Martín, J. A., Guilló, S., Gatti, M., Rodríguez, P., Baeza, P., Fernández, J. L. y López, J. (2019). LGTBfobia en las aulas 2019. COGAM. https://cogam.es/wp-content/uploads/2020/04/LGBTfobia-en-las-aulas-2019.pdf

Pérez, P. D., Fernández, M., García, E. y Guerra, P. (2021). Acoso escolar y autoconcepto en personas trans. Revista de Investigación Educativa, 39(1), 189-208. https://doi.org/10.6018/rie.420001

Pichado, J. I. y De Stéfano, M. (2015). El alumnado de secundaria ante la diversidad sexual. En J. I. Pichardo y M. de Stéfano (Eds.), Diversidad sexual y convivencia (pp. 63-81). Universidad Complutense de Madrid. https://eprints.ucm.es/id/eprint/34926/1/Diversidad_y_convivencia_2015_FINAL.pdf

Puche, L., Moreno, E. y Pichardo, J. I. (2013). Adolescentes transexuales en las aulas. Aproximación cualitativa y propuestas de intervención desde la perspectiva antropológica. En 0. Moreno y L. Puche (Eds.), Transexualidad, adolescencias y educación. Miradas multidisciplinares (pp. 189-265). Editorial Egales. 